\title{
Adaptación psicométrica del test para evaluar procesos de simplificación fonológica (Teprosif-R)
}

\author{
Érika Albarracín, Pamela Carranza \& Elisa Meléndez \\ Centro Peruano de Audición, Lenguaje y Aprendizaje
}

Recibido: 18 de abril del 2013 / Aprobado: 27 de junio del 2013

El objetivo del estudio fue adaptar psicométricamente el test para evaluar procesos de simplificación fonológica (Teprosif-R) elaborado para identificar los procesos de simplificación fonológica de niños de 3 a 6 años. El Teprosif-R adaptado se aplicó a 448 niños y niñas, de 3 a 6 años, de instituciones educativas estatales y privadas. Los resultados demostraron que los puntajes de adaptación $(0,94)$ eran confiables. Asimismo, se llegó a la conclusión de que dicha prueba posee validez de contenido, evaluada a través del juicio de expertos. Se determinaron los niveles de dificultad de los reactivos y se obtuvieron normas en percentiles para interpretar los niveles de desempeño según el total de procesos de simplificación fonológica en cada rango de edad.

desarrollo fonológico / procesos de simplificación fonológica / confiabilidad / validez

Psychometric adaptation test to evaluate the phonological simplification processes (Teprosif-R)

The aim of this study was to adapt psychometrically Test to Evaluate Phonological Simplification Processes (Teprosif-R) prepared to identify phonological simplification processes of children 3 to 6 years. Applied Teprosif-R adapted to 448 boys and girls, from 3 to 6 years of public and private educational institutions. Results showed that adaptation scores (0.94) were reliable. It also is concluded that this test has content validity, assessed through the judgment of experts. It was determined the levels of difficulty of the reagents and standards was obtained in percentiles norms for interpreting levels of performance according to the total of phonological simplification processes in each age range.

phonological development / phonological simplification processes / reliability / validity

Correo electrónico: icpe@cpal.edu.pe 


\section{INTRODUCCIÓN}

El lenguaje es un sistema complejo integrado por distintos módulos o componentes (Acosta \& Moreno, 2001), los cuales se pueden agrupar en formales (sintaxis, morfología y fonología) de contenido (semántico) y de uso (pragmático). Asimismo, lo integran la prosodia o entonación, que puede modificar el significado literal de las palabras, de las frases y del discurso.

Todo lenguaje está organizado como una jerarquía de estructuras: discurso, frases, palabras, morfemas y fonemas (Acosta \& Moreno, 2001). Cada nivel de la jerarquía está gobernado por su propio sistema de reglas, como en el caso de las reglas fonológicas que especifican que en un determinado lenguaje existen fonemas que pueden ir juntos, existiendo otros casos donde no se puede cumplir la regla anteriormente mencionada. Para lograr un desarrollo óptimo del lenguaje oral es indispensable ampliar la base comunicativa hacia lo significativo y lo expresivo, buscando la coherencia de sus componentes.

\section{Componentes del lenguaje}

\section{El componente semántico}

Según Santiuste y Beltrán (1998), la semántica analiza el contenido o significado de las palabras. En este aspecto, el niño se ve ampliamente influido por las interacciones sociales y culturales del medio. Por su parte, Acosta et al. (2001) señala que la semántica se centra en el significado de las palabras y sus combinaciones. De aquí se concluye que la semántica es «lo que tiene significado»; su finalidad es establecer el significado de los signos y su influencia en lo que la gente hace y dice. Es el componente que se relaciona, por tanto, con el significado e incluye el conocimiento de las categorías conceptuales del lenguaje, de las palabras y expresiones que se usan en conceptos (léxico).

Santiuste \& Beltrán (1998) señalan que la morfología se dedica al estudio de las unidades más pequeñas del lenguaje que tienen sentido, así como las reglas que determinan la estructura de las palabras y de su variabilidad. También se refiere a la estructura de las palabras, las cuales se pueden descomponer en partes más pequeñas denominadas afijos: prefijos y sufijos. Como lo indica su nombre, la morfología se encarga de estudiar la estructura interna de las palabras desde la perspectiva de sus formas. Dockrell y Mc. Shine (1997) la explican como un conjunto de principios que determinan cómo se pueden combinar las palabras de una forma gramatical.

La importancia de la sintaxis en la comunicación se basa en que orienta la construcción adecuada de las oraciones, dando como resultado una expresión oral coherente. En términos simplificados, la sintaxis es el orden y la estructura de las palabras y frases dentro de 
la gramática. Incluye el dominio de las relaciones entre las palabras dentro de las oraciones y de cómo expresar estas relaciones. Cada elemento sintáctico constituye una unidad funcional.

\section{El componente pragmático}

Se encarga del estudio del lenguaje en contextos sociales, considerando la intencionalidad comunicativa del hablante y del empleo que hace del lenguaje para su comunicación.

Por lo tanto, presta especial interés a las reglas que gobiernan el uso social del lenguaje, considerando tres aspectos en su estudio: las intenciones comunicativas, la organización del discurso conversacional y las presuposiciones en contextos conversacionales y narrativos.

\section{El componente fonético-fonológico}

Se relaciona con el estudio del habla y analiza sus dificultades de orden articulatorio (fonética), como de orden fonológico.

Por lo tanto, debemos distinguir fonología de fonética. La fonología se encarga del estudio de los sonidos de la expresión lingüística desde un punto de vista funcional y abstracto. Martínez Celdrán (1989; en Acosta et al., 2001), menciona que su «estudio se lleva mediante la utilización de criterios estrictamente lingüísticos», es decir, estudia la organización de los sonidos durante la articulación del habla espontánea, valiéndose de sus características articu- latorias y de los distintos contextos en los que puede aparecer. Por su parte, la fonética estudia la producción sonora y procura recoger información sobre las propiedades fisiológicas y físicas de los sonidos de habla.

Para Acosta et al. (2001), la fonología se interesa por el estudio de la organización de los sonidos en un sistema valiéndose de sus caracteres articulatorios y de la distribución o suma de los contextos en que pueden aparecer. Lo que indica que la fonología intenta entender la influencia que los sonidos tienen unos sobre otros, dando sentido a los datos fonéticos y analizando elementos y reconociendo el mismo sonido.

\section{DesARROLLO FONOLÓGICO}

Para Bosch (2004), la evaluación de los procesos de simplificación fonológica es un área del lenguaje que permite identificar problemas de comunicación $\mathrm{y}$, a su vez, identificar problemas del aprendizaje de la lectoescritura.

Sin embargo, resulta básico entender el proceso de adquisición de las habilidades fonológicas que definan el marco en que debe ubicarse el test de evaluación en estudio.

Cervera e Ygual (2003) dividen la adquisición de las habilidades fonológicas en 4 periodos.

El primer periodo corresponde a una etapa prelingüística en la que el niño no emite palabras. Podríamos decir que es una etapa sin fonología, condicionada 
por la maduración de los sistemas biológicos de la percepción y producción del habla. El segundo periodo corresponde a la etapa lingüística de la holofrase y dura entre 6 meses y 1 año. Durante este periodo, el niño se comunica lingüísticamente con enunciados de palabras aisladas. Desde el punto de vista de la fonología, la etapa se conoce como «del consonantismo mínimo» o «fonología de las primeras 50 palabras». El tercer periodo de desarrollo fonológico comienza cuando el niño realiza las primeras combinaciones de palabras (combinaciones eje-abierta o gramática combinatoria) y se prolonga durante todo el periodo de adquisición de la frase simple. Se puede localizar entre los 18 o 24 meses y los 4 años. Se denomina «periodo de la fonología del morfema simple» y es la etapa en la que un niño de desarrollo normal realiza mayores progresos en cuanto a los códigos lingüísticos. Dominan la etapa los denominados «procesos de simplificación del habla» o «procesos fonológicos». Se trata de un conjunto de reglas de reducción de la complejidad de la forma de las palabras para permitir que el niño las use en su léxico expresivo, a pesar de ser incapaz de reproducir exactamente la forma adulta. Estos procesos inicialmente son muy reductores, afectan a la inteligibilidad y asemejan las palabras al consonantismo mínimo de la etapa anterior, pero progresivamente se hacen más sofisticados, hasta su total desaparición. La cuarta etapa, o «de culminación», comprende desde los 4 a los 6 años y supone el cierre de las habilidades de procesamiento del habla: el niño, al final del periodo, puede identificar y reproducir cualquier secuencia de fonemas habitual en la lengua, conocida o no, con significado o sin él. Durante este periodo todavía pueden ser normales algunos procesos de simplificación del habla que atañen a segmentos muy concretos y de difícil realización. Durante este periodo, y ligado tanto al desarrollo fonológico como al aprendizaje de la lectura y escritura, también se desarrollan las habilidades metafonológicas.

\section{PROCESOS DE SIMPLIFICACIÓN FONOLÓGICA}

Al elaborar el Teprosif-R, Pavez, Maggiolo y Coloma (2008) consideran 8 procesos relacionados con la estructura de la sílaba y de la palabra, 9 de asimilación y 16 de sustitución. Los primeros se identifican con la letra $\mathrm{E}$, los segundos con la A y los terceros con la S. Sin embargo, el análisis que hemos realizado con la ayuda de los asesores metodológicos y de contenido nos ha llevado a incluir un proceso más, denominado «asimilación velar».

a) Procesos relacionados con la estructura de la palabra.- Son procedimientos mediante los cuales el niño simplifica las sílabas transformándolas en estructuras del tipo consonante + vocal (CV) o simplifi- 
ca la estructura métrica o rítmica de la palabra para facilitar su emisión. Los procesos relacionados con la estructura de la sílaba y la palabra pueden clasificarse en los siguientes tipos: reducción del grupo consonántico, reducción del diptongo, omisión de la consonante trabante o coda silábica, coalescencia, omisión de elementos átonos, omisión de la sílaba tónica o de alguno de sus constituyentes, adición de fonemas o sílabas (epéntesis), inversión de fonemas o sílabas (metátesis).

b) Procesos de asimilación.- Son procedimientos mediante los cuales se cambia un fonema para hacerlo igual o semejante a otro presente en la palabra modelo o en la emitida por el niño. Existen asimilaciones idénticas y asimilaciones en que un fonema se asemeja a otro. Pueden hacerse semejantes en la zona de articulación y en la resonancia. También es posible que fonemas no líquidos se asimilen a los líquidos (/r/, / $\overline{\mathrm{r}} / \mathrm{o} / \mathrm{l} /)$, que un fonema vocálico se asimile a otro vocálico (en la zona o el grado de abertura) y que una sílaba se haga igual a otra, presente en la palabra. Pueden clasificarse en los siguientes tipos: asimilación idéntica, asimilación labial, asimilación dental, asimilación alveolar, asimilación palatal, asimilación velar, asimilación a fonemas líquidos, asimilación nasal, asimilación vocálica, asimilación silábica o duplicación. c) Procesos de sustitución.- Son procedimientos para simplificar la palabra donde se sustituyen fonemas pertenecientes a una clase por miembros de otra clase; o fonemas de una misma clase entre sí, como ocurre en los líquidos y en los fricativos. Los procesos de sustitución pueden afectar a los fonemas que traban la sílaba (coda silábica), como ocurre en la aspiración del fonema trabante de sílaba. Además, estos procesos pueden ocurrir en una zona de articulación dentro de la cavidad oral, presentándose una posteriorización, frontalización y labialización de consonantes. Asimismo, se presentan procesos según el modo de articulación, dando lugar a la oclusivización o pérdida del rasgo fricativo, fricativización de fonemas oclusivos o africados y sustitución de fonemas fricativos entre sí.

A la vez, existen procesos de sustitución, según fonación o cualidad articulatoria, los cuales se producen por la sonorización de consonantes, el ensordecimiento o la pérdida de sonoridad o afonización de consonantes. Por otra parte, están los procesos que afectan el rasgo fundamental sustituyendo fonemas líquidos/no líquidos, dando lugar a la semiconsonantización de fonemas líquidos, sustitución de fonemas líquidos entre sí, sustitución de fonemas líquidos por no líquidos orales, sustitución de fonemas consonán- 
ticos no líquidos orales o vocálicos orales por fonemas líquidos.

Finalmente, se encuentran los procesos de sustitución que afectan la resonancia suplementaria, mostrándose una nasalización de fonemas y oralización de consonantes nasales. Por último, están los procesos que afectan a los fonemas vocálicos, denominados sustitución de vocales o disimilación.

Para analizar el buen desempeño del desarrollo fonológico de los niños se hace necesaria la evaluación de los procesos de simplificación fonológica, que es un área del lenguaje que permite identificar los problemas de comunicación referidos al tipo de simplificaciones de habla presentes en producciones verbales relativas a la estructura de la palabra, sustituciones y asimilaciones y a su vez identificar problemas del aprendizaje de la lectoescritura.

En el ámbito mundial existen instrumentos indicados para el estudio de estos procesos, entre ellos cabe mencionar la prueba de desarrollo fonológico infantil de Bosch (1983), que es una prueba de screening, dado que su finalidad primordial es detectar niños cuyo desarrollo fonológico difiere del que es normativo; el test para evaluar procesos de simplificación fonológica (Teprosif) de Maggiolo y Pavez (2000), creado para detectar a niños con trastorno fonológico; y el test para evaluar procesos de simplificación fonológica versión revisada (Teprosif-R) (Maggiolo, Pa- vez \& Coloma, 2008), cuyos objetivos son identificar los procesos con los que los niños de 3 a 6 años simplifican la producción fonológica de sus palabras y entregar normas para establecer si la cantidad de procesos que presentan corresponde a lo esperable a su edad.

Este último instrumento es el que se considera adaptable a nuestra realidad, debido a que fue creado en un país geográficamente situado cerca del Perú, pero con ciertas características de idioma castellano diferentes, motivo por el cual se ha realizado todo un proceso de adaptación, de acuerdo con las características lingüísticas de nuestro medio social.

La meta planteada en este estudio fue contar, en el campo de la investigación del lenguaje de nuestro país, con un instrumento que sirva para evaluar los procesos de simplificación fonológica de niños de 3 a 6 años, para lo cual se realizó la adaptación lingüística y pictórica del Teprosif-R, así como la estimación de su validez y confiabilidad y el establecimiento de las normas psicométricas para la interpretación de las puntuaciones directas que los niños evaluados logran en el test.

OBjetivos

\section{Objetivo general}

Adaptar psicométricamente el test para evaluar procesos de simplificación fonológica (Teprosif-R) en niños y niñas 
de 3 a 6 años pertenecientes a instituciones educativas privadas y estatales de Lima metropolitana.

\section{Objetivos específicos}

Entre los objetivos específicos se encuentran los siguientes:

- Adaptar lingüística y pictóricamente el Teprosif-R en la población de referencia.

- Obtener evidencias de validez de contenido del Teprosif-R adaptado a través del juicio de expertos.

- Obtener evidencia de la validez de constructo del Teprosif-R adaptado a través del análisis de la intercorrelación de sus componentes así como su relación con la edad.

- Estimar empíricamente la confiabilidad del Teprosif-R adaptado empleando el método de consistencia interna.

- Determinar los niveles de dificultad de los reactivos del Teprosif-R adaptado.

- Elaborar normas para la interpretación del Teprosif-R adaptado.

\section{MÉTOdo}

\section{Diseño de investigación}

La presente investigación corresponde a un estudio descriptivo y psicométrico (Sánchez \& Reyes, 2006; Alarcón,
2008; Hernández, Fernández \& Baptista, 2010). Se analizan las propiedades psicométricas de test para evaluar procesos de simplificación fonológica (Teprosif-R) adaptado en niñas de 3 a 6 años pertenecientes a instituciones educativas privadas y estatales de Lima metropolitana.

\section{Población y muestra de la} investigación

La población está compuesta por niños y niñas de 3 a 6 años, estudiantes de nivel inicial y primario de instituciones educativas estatales y privadas de Lima metropolitana.

El tipo de muestreo que se utilizó es el probabilístico por conglomerados polietápico, definiéndose como primera unidad de análisis a las unidades de gestión educativa local de Lima metropolitana (UGEL); como segunda unidad de análisis se seleccionaron 23 colegios, 10 particulares y 13 estatales. Posteriormente, en la tercera unidad de análisis se eligieron las aulas $\mathrm{y}$, finalmente, en la cuarta unidad de análisis se escogieron aleatoriamente los sujetos de la muestra, empleándose una estratificación uniforme por sexo, grado y tipo de colegio. En el momento de la selección se consideró como único criterio de exclusión que los niños tuvieran retraso o dificultad de tipo fonológico (véase la tabla 1). 
Tabla 1

Relación de instituciones educativas participantes del estudio

\begin{tabular}{|c|c|c|c|c|}
\hline UGEL & $\begin{array}{l}\text { Instituciones } \\
\text { educativas }\end{array}$ & $f$ & $\%$ & Total \\
\hline \multirow{2}{*}{1} & 1 & 23 & 5,13 & \multirow{2}{*}{55} \\
\hline & 2 & 32 & 7,14 & \\
\hline \multirow{3}{*}{2} & 3 & 8 & 1,79 & \multirow{3}{*}{64} \\
\hline & 4 & 24 & 5,36 & \\
\hline & 5 & 32 & 7,14 & \\
\hline \multirow{4}{*}{3} & 6 & 8 & 1,79 & \multirow{4}{*}{64} \\
\hline & 7 & 24 & 5,36 & \\
\hline & 8 & 8 & 1,79 & \\
\hline & 9 & 24 & 5,36 & \\
\hline \multirow{4}{*}{4} & 10 & 8 & 1,79 & \multirow{4}{*}{64} \\
\hline & 11 & 24 & 5,36 & \\
\hline & 12 & 8 & 1,79 & \\
\hline & 13 & 24 & 5,36 & \\
\hline \multirow{3}{*}{5} & 14 & 8 & 1,79 & \multirow{3}{*}{64} \\
\hline & 15 & 24 & 5,36 & \\
\hline & 16 & 32 & 7,14 & \\
\hline \multirow{4}{*}{6} & 17 & 24 & 5,36 & \multirow{4}{*}{64} \\
\hline & 18 & 8 & 1,79 & \\
\hline & 19 & 24 & 5,36 & \\
\hline & 20 & 8 & 1,79 & \\
\hline \multirow{3}{*}{7} & 21 & 28 & 6,25 & \multirow{3}{*}{73} \\
\hline & 22 & 13 & 2,90 & \\
\hline & 23 & 32 & 7,14 & \\
\hline
\end{tabular}

$N=448$

El tamaño de la muestra se calculó para estimar los parámetros poblacionales con un nivel de confianza del $95 \%$ y un margen de error $+/-5 \%$; a la cantidad resultante se le complementó por facilidades halladas en la aplicación a un número determinado de sujetos, de manera tal que el tamaño final de la muestra de estudio fue de 448 niños y niñas de 3 a 6 años de Lima metropolitana, canti- 
dad que contribuye a precisar mejor los parámetros poblacionales, vale decir, el promedio de la población y varianza poblacional (véase la tabla 2 ).

Tabla 2

Distribución de muestra según edad y sexo

\begin{tabular}{lrrrr} 
& \multicolumn{2}{c}{ Masculino } & \multicolumn{2}{c}{ Femenino } \\
escolar & f & $\%$ & $\mathbf{f}$ & $\%$ \\
\cline { 2 - 5 } 3 años & 56 & 50 & 56 & 50 \\
4 años & 56 & 50 & 56 & 50 \\
5 años & 56 & 50 & 56 & 50 \\
6 años & 56 & 50 & 56 & 50 \\
\hline $\mathrm{N}=448$ & & & &
\end{tabular}

Instrumento de recolección de datos

El instrumento utilizado fue el Teprosif-R, el cual fue creado y normado en Chile el año 2008, por sus autoras, María Mercedes Pavez, Mariangela Maggiolo y Carmen Julia Coloma, su segunda edición fue realizada por la Universidad Católica de Chile (Maggiolo, Pavez \& Coloma, 2008).

Fue creado para evaluar los procesos de simplificación fonológica en diversas palabras de diferente complejidad. La aplicación del test se realiza en forma individual a niños con edades comprendidas entre los 3 y 6 años de edad y tiene una duración de entre 10 y 15 minutos, en función de la edad.

El material incluye un manual de aplicación, un set de 37 láminas, una hoja de respuestas, una hoja de análisis y un esquema para orientar el análisis de las respuestas.

La confiabilidad del Teprosif-R se realizó mediante el coeficiente alfa de Cronbach y el valor obtenido fue 0.90 . Según lo anterior, el Teprosif-R muestra un alto índice de confiabilidad. Las evidencias de validez concurrente se obtuvieron a través del estudio, en primer término, de una comparación entre el Teprosif-R y el Teprosif, con el objetivo de saber si existía relación entre los resultados obtenidos entre ambas pruebas. Se seleccionaron aleatoriamente 357 niños de la muestra (evaluados con la versión revisada) y se les aplicó el Teprosif original. La cantidad total de PSF obtenidos en las ambas evaluaciones se analizó con la prueba de Pearson, encontrándose que existe correlación significativa entre los dos instrumentos $(\mathrm{r}=0.92, \mathrm{p}<.001)$; es decir, los resultados obtenidos en el Teprosif-R se relacionan significativamente con el test original, lo que contribuye a evidenciar validez de la medida. Posteriormente, en los 620 niños de la muestra se analizó si existía relación entre los resultados de Teprosif-R y la edad expresada en meses. Para ello se aplicó también la prueba de Pearson y se constató una correlación negativa y estadísticamente significativa entre am- 
bas variables $(\mathrm{r}=64, \mathrm{p}<.001)$; así, con esta información se avala la validez del test, pues el aumento de la edad se relaciona de modo significativo con la disminución de PSF. Luego, con el objetivo de verificar si el Teprosif-R discrimina entre los rangos de edad se comparó la cantidad total de PSF de los distintos grupos etarios mediante un Anova. Un procedimiento similar se realizó con cada tipo de PSF, encontrándose una disminución significativa de los PSF a medida que se avanza en edad.

\section{Procedimiento de recolección de datos}

El proceso de adaptación del Teprosif-R a la realidad de los niños de Lima metropolitana se realizó mediante un estudio piloto del test original, aplicándose el instrumento a una muestra de 10 niños. Luego, se adaptaron lingüísticamente los ítems 2, 6, 9, 11, 21, 28 y 32 , respetando la estructura silábica, la acentuación y el número de sílabas. Igualmente, se modificaron gráficamente los estímulos del ejemplo $2 \mathrm{y}$ las láminas 1, 2, 5, 6, 10, 11, 13, 14, 15, $17,21,23,25,27,28,32,33$ y 37 , a fin de mejorar la calidad del gráfico y su percepción visual. Asimismo, se adicionaron las láminas 38, 39 y 40, con el objetivo de evaluar el fonema $/ \tilde{\mathrm{n}} / \mathrm{y}$ los grupos consonánticos $/ \mathrm{gl} / \mathrm{y} / \mathrm{dr} /$, que no eran considerados en el test original. Estas variaciones se realizaron bajo el asesoramiento de un lingüista y un experto en adaptaciones psicométricas.
Posteriormente, se validó el Teprosif- $\mathrm{R}$ adaptado lingüística y gráficamente a través del juicio de expertos, por el método de calificación de ítems. Luego, se realizó la administración del instrumento adaptado lingüísticamente a la población de estudio, considerando las condiciones psicométricas usuales en la medición y atributos psicológicos o psicolingüísticos. Así por ejemplo, se capacitó a 6 psicólogos en la aplicación del test.

Se efectuó la calificación manual de los protocolos de respuesta de una manera bastante cuidadosa y rigurosa. Se construyó la base de datos en Excel, la misma que fue sometida a análisis estadístico con el Statistical Package for the Social Sciences 19 (SPSS), de acuerdo con los propósitos de estudio, para obtener los principales estadísticos descriptivos e inferenciales.

\section{Resultados}

Análisis de la confiabilidad

La confiabilidad del Teprosif-R adaptado se analizó mediante el coeficiente alfa de Cronbach, siendo el valor obtenido 0.94. Según lo anterior, el Teprosif-R evidencia un alto índice de confiabilidad.

Se analizó la relación de los ítems con el puntaje total de la prueba mediante la prueba de Pearson, los resultados mostraron que todos los ítems se correlacionan significativamente con 
Tabla 3

Correlación de los ítems con el puntaje total

\begin{tabular}{cc|cc}
\hline Ítem & $\begin{array}{c}\text { Correlación } \\
\text { ítem-total }\end{array}$ & Ítem & $\begin{array}{c}\text { Correlación } \\
\text { ítem-total }\end{array}$ \\
\hline 1 & $0.56^{* *}$ & 21 & $0.58^{* *}$ \\
2 & $0.35^{* *}$ & 22 & $0.58^{* *}$ \\
3 & $0.67^{* *}$ & 23 & $0.53^{* *}$ \\
4 & $0.64^{* *}$ & 24 & $0.45^{* *}$ \\
5 & $0.57^{* *}$ & 25 & $0.67^{* *}$ \\
6 & $0.51^{* *}$ & 26 & $0.43^{* *}$ \\
7 & $0.64^{* *}$ & 27 & $0.58^{* *}$ \\
8 & $0.67^{* *}$ & 28 & $0.44^{* *}$ \\
9 & $0.64^{* *}$ & 29 & $0.67^{* *}$ \\
10 & $0.64^{* *}$ & 30 & $0.48^{* *}$ \\
11 & $0.66^{* *}$ & 31 & $0.63^{* *}$ \\
12 & $0.70^{* *}$ & 32 & $0.61^{* *}$ \\
13 & $0.49^{* *}$ & 33 & $0.51^{* *}$ \\
14 & $0.69^{* *}$ & 34 & $0.47^{* *}$ \\
15 & $0.50^{* *}$ & 35 & $0.49^{* *}$ \\
16 & $0.63^{* *}$ & 36 & $0.57^{* *}$ \\
17 & $0.60^{* *}$ & 37 & $0.41^{* *}$ \\
18 & $0.54^{* *}$ & 38 & $0.30^{* *}$ \\
19 & $0.42^{* *}$ & 39 & $0.59^{* *}$ \\
20 & $0.71^{* *}$ & 40 & $0.58^{* *}$ \\
\hline
\end{tabular}

** $p<0.01$

el puntaje total del test, lo que avala la consistencia interna de la prueba (véase la tabla 3).

Posteriormente, con el propósito de conocer el nivel de dificultad del Teprosif-R, se analizó también el grado de dificultad de los ítems. Los resultados arrojan que los ítems varían en grado de dificultad entre los 3 y 4 años. En cambio, a los 5 y 6 años, se incrementa la cantidad de ítems fáciles. Al sintetizar la información, señalando el porcentaje total de ítems con los distintos niveles de dificultad en cada rango etario, se corrobora que la prueba es más fácil a medida que aumenta la edad de los niños (véase la tabla 4).

La disminución del grado de dificultad de la prueba según aumenta la edad confirma su sensibilidad y validez para indicar la disminución de PSF en el desarrollo fonológico de los niños. 
Tabla 4

Dificultad de los ítems según el porcentaje de niños que emiten procesos en cada rango de edad

\begin{tabular}{|c|c|c|c|c|c|c|c|c|}
\hline \multirow{2}{*}{$\begin{array}{c}\begin{array}{c}\text { Rango } \\
\text { de edad }\end{array} \\
\text { Ítem }\end{array}$} & \multicolumn{2}{|c|}{$\begin{array}{c}3 \text { años } \\
n=112\end{array}$} & \multicolumn{2}{|c|}{$\begin{array}{c}4 \text { años } \\
n=112\end{array}$} & \multicolumn{2}{|c|}{$\begin{array}{c}5 \text { años } \\
n=112\end{array}$} & \multicolumn{2}{|c|}{$\begin{array}{c}6 \text { años } \\
n=112\end{array}$} \\
\hline & $\%$ & Dificultad & $\%$ & Dificultad & $\%$ & Dificultad & $\%$ & Dificultad \\
\hline 1 & 31,3 & $M$ & 21,4 & $\mathrm{~F}$ & 3,6 & $F$ & 4,5 & $\mathrm{~F}$ \\
\hline 2 & 49,1 & $M$ & 52,7 & $\mathrm{D}$ & 31,3 & $M$ & 23,2 & $\mathrm{~F}$ \\
\hline 3 & 47,3 & $M$ & 24,1 & $F$ & 11,6 & $\mathrm{~F}$ & 2,7 & $F$ \\
\hline 4 & 48,2 & $M$ & 32,1 & $M$ & 13,5 & $\mathrm{~F}$ & 7,1 & $\mathrm{~F}$ \\
\hline 5 & 83,0 & MD & 86,6 & $M D$ & 75,0 & D & 50,9 & M \\
\hline 6 & 25,0 & $\mathrm{~F}$ & 17,0 & $\mathrm{~F}$ & 3,6 & $\mathrm{~F}$ & 1,8 & $\mathrm{~F}$ \\
\hline 7 & 67,0 & D & 48,2 & M & 27,7 & $M$ & 14,3 & $\mathrm{~F}$ \\
\hline 8 & 89,3 & MD & 80,4 & MD & 61,6 & $D$ & 46,4 & $M$ \\
\hline 9 & 85,7 & MD & 83,9 & MD & 61,6 & $\mathrm{D}$ & 42,9 & $M$ \\
\hline 10 & 64,3 & $D$ & 43,8 & $M$ & 26,8 & $M$ & 17,0 & $\mathrm{~F}$ \\
\hline 11 & 43,8 & $M$ & 29,5 & M & 12,5 & $\mathrm{~F}$ & 13,4 & $F$ \\
\hline 12 & 66,1 & $D$ & 47,3 & $M$ & 26,8 & $M$ & 15,2 & $\mathrm{~F}$ \\
\hline 13 & 21,4 & $F$ & 25,9 & $M$ & 4,5 & $F$ & 3,6 & $F$ \\
\hline 14 & 67,0 & $\mathrm{D}$ & 43,8 & $M$ & 14,3 & $\mathrm{~F}$ & 9,8 & $\mathrm{~F}$ \\
\hline 15 & 41,1 & M & 21,4 & $\mathrm{~F}$ & 8,0 & $\mathrm{~F}$ & 2,7 & $\mathrm{~F}$ \\
\hline 16 & 40,2 & $M$ & 31,3 & $M$ & 5,4 & $\mathrm{~F}$ & 4,5 & $\mathrm{~F}$ \\
\hline 17 & 35,7 & $M$ & 20,5 & $\mathrm{~F}$ & 6,3 & $\mathrm{~F}$ & 1,8 & $\mathrm{~F}$ \\
\hline 18 & 25,0 & $\mathrm{~F}$ & 17,0 & $\mathrm{~F}$ & 2,7 & $\mathrm{~F}$ & 4,5 & $\mathrm{~F}$ \\
\hline 19 & 13,4 & $\mathrm{~F}$ & 6,3 & $\mathrm{~F}$ & 2,7 & $\mathrm{~F}$ & 0,0 & $\mathrm{~F}$ \\
\hline 20 & 71,4 & $D$ & 44,6 & $M$ & 23,2 & $\mathrm{~F}$ & 12,5 & $\mathrm{~F}$ \\
\hline 21 & 65,2 & $D$ & 48,2 & $M$ & 20,5 & $\mathrm{~F}$ & 12,5 & $\mathrm{~F}$ \\
\hline 22 & 53,6 & $D$ & 34,8 & $M$ & 16,1 & $\mathrm{~F}$ & 7,1 & $\mathrm{~F}$ \\
\hline 23 & 25,9 & $M$ & 16,1 & $\mathrm{~F}$ & 5,4 & $\mathrm{~F}$ & 2,7 & $\mathrm{~F}$ \\
\hline 24 & 32,1 & $M$ & 30,4 & $M$ & 7,1 & $F$ & 8,9 & $F$ \\
\hline 25 & 50,9 & $M$ & 37,5 & $M$ & 10,7 & $F$ & 6,3 & $F$ \\
\hline 26 & 10,7 & $\mathrm{~F}$ & 6,3 & $\mathrm{~F}$ & 0,0 & $\mathrm{~F}$ & 0,9 & $\mathrm{~F}$ \\
\hline 27 & 45,5 & $M$ & 29,5 & $M$ & 8,9 & $\mathrm{~F}$ & 3,6 & $\mathrm{~F}$ \\
\hline 28 & 17,0 & $\mathrm{~F}$ & 9,8 & $\mathrm{~F}$ & 3,6 & $\mathrm{~F}$ & 3,6 & $\mathrm{~F}$ \\
\hline
\end{tabular}


(continuación)

\begin{tabular}{|c|c|c|c|c|c|c|c|c|}
\hline \multirow{2}{*}{$\begin{array}{c}\begin{array}{c}\text { Rango } \\
\text { de edad }\end{array} \\
\text { Ítem }\end{array}$} & \multicolumn{2}{|c|}{$\begin{array}{c}3 \text { años } \\
n=112\end{array}$} & \multicolumn{2}{|c|}{$\begin{array}{c}4 \text { años } \\
n=112\end{array}$} & \multicolumn{2}{|c|}{$\begin{array}{c}5 \text { años } \\
n=112\end{array}$} & \multicolumn{2}{|c|}{$\begin{array}{c}6 \text { años } \\
n=112\end{array}$} \\
\hline & $\%$ & Dificultad & $\%$ & Dificultad & $\%$ & Dificultad & $\%$ & Dificultad \\
\hline 29 & 42,0 & $M$ & 23,2 & $\mathrm{~F}$ & 4,5 & $\mathrm{~F}$ & 5,4 & $\mathrm{~F}$ \\
\hline 30 & 54,5 & $\mathrm{D}$ & 42,0 & $M$ & 17,9 & $\mathrm{~F}$ & 13,4 & $\mathrm{~F}$ \\
\hline 31 & 31,3 & $M$ & 21,4 & $\mathrm{~F}$ & 3,6 & $\mathrm{~F}$ & 4,5 & $\mathrm{~F}$ \\
\hline 32 & 35,7 & $M$ & 21,4 & $\mathrm{~F}$ & 2,7 & $\mathrm{~F}$ & 4,5 & $\mathrm{~F}$ \\
\hline 33 & 63,4 & $\mathrm{D}$ & 46,4 & $\mathrm{M}$ & 24,1 & $\mathrm{~F}$ & 25,9 & M \\
\hline 34 & 27,7 & $M$ & 10,7 & $\mathrm{~F}$ & 0,0 & $\mathrm{~F}$ & 2,7 & $\mathrm{~F}$ \\
\hline 35 & 87,5 & MD & 70,5 & MD & 49,1 & $M$ & 48,2 & $M$ \\
\hline 36 & 38,4 & $M$ & 28,6 & $M$ & 5,4 & $\mathrm{~F}$ & 3,6 & $\mathrm{~F}$ \\
\hline 37 & 42,9 & M & 20,5 & $\mathrm{~F}$ & 10,7 & $\mathrm{~F}$ & 6,3 & $F$ \\
\hline 38 & 8,0 & $F$ & 3,6 & $\mathrm{~F}$ & 0,0 & $\mathrm{~F}$ & 0,9 & $\mathrm{~F}$ \\
\hline 39 & 52,7 & $D$ & 46,6 & $M$ & 17,0 & $\mathrm{~F}$ & 8,9 & $\mathrm{~F}$ \\
\hline 40 & 87,5 & MD & 68,8 & $\mathrm{D}$ & 45,5 & $\mathrm{~F}$ & 29,5 & $M$ \\
\hline
\end{tabular}

Nota:Nivelesdedificultad,Fácil(F)=10\%a25\%deniñossimplificanlapalabra; Mediano(M)=26\%a50\%deniñossimplificanla palabra; Difícil (D) $=51 \%$ a $75 \%$ de niños simplifican la palabra; Muy Difícil (MD) $=76 \%$ a $100 \%$ de niños simplifican la palabra .

\section{EVIDENCIAS DE VALIDEZ}

Los resultados de la validación del Teprosif-R adaptado lingüística y gráficamente se obtuvieron a través del juicio de expertos. Los resultados muestran que la aprobación global de la adaptación del Teprosif-R adaptado superó el $75 \%$ de acuerdo entre los jueces, por lo tanto el instrumento presenta evidencias de validez del contenido.

En la tabla 5 se muestran los resultados del índice de acuerdos frente al jui- cio de expertos. Tanto en el criterio 1, referido a la congruencia entre los objetivos de la investigación y el instrumento; el criterio 2, concerniente a la coherencia entre instrucciones y consignas; el criterio 3, alusivo a la organización lógica; el criterio 5 , perteneciente a la coherencia interna entre variables, indicadores e ítems, se obtuvo un $100 \%$ de acuerdo. Solo en el caso del criterio 4 , referente a si el lenguaje es acorde al público objetivo, se obtuvo un $86 \%$ de aprobación. 
Tabla 5

Índices de acuerdo por el método de criterio de jueces

\begin{tabular}{llc}
\hline Criterio & $\begin{array}{c}\text { Porcentaje } \\
\text { de acuerdos }\end{array}$ \\
\hline 1. Si el instrumento contribuye a lograr el objetivo de la investigación. & 100 \\
2. Si las instrucciones son fáciles de seguir. & 100 \\
3. Si el instrumento está organizado en forma lógica. & 100 \\
4. Si el lenguaje utilizado es apropiado para el público al que va dirigido. & 86 \\
5. Si existe coherencia entre las variables, indicadores e ítems. & 100 \\
\hline
\end{tabular}

$\mathrm{N}=7$ jueces.

Para validar la versión peruana del Teprosif-R se analizó si existía relación entre los resultados del Teprosif-R adaptado y la edad expresada en meses. Para ello se aplicó la prueba de Pearson y se constató una correlación negativa $\mathrm{y}$ estadísticamente significativa entre ambas variables $(\mathrm{r}-0.6240, \mathrm{p}=0.000)$. Así, a partir de este se obtienen evidencias de la validez del test, pues el aumento de la edad se relaciona de modo significativo con la disminución del PSF, siendo la media de PSF 27,45 para 3 años a 3 años 11 meses, el promedio de PSF 19,21 para 4 años a 4 años 11 meses, el promedio de PSF es de 8,67 para 5 años a 5 años 11 meses y el promedio de PSF 5,88 para 6 años a 6 años 11 meses.

Luego, con el objetivo de verificar si el Teprosif-R adaptado discrimina entre los rangos de edad, como evidencia de validez de constructo, se comparó esta- dísticamente la cantidad total del PSF de los distintos grupos etarios mediante un Anova. Un procedimiento similar se realizó con cada tipo de PSF.

En la tabla 6 se advierte, según el resultado estadísticamente significativo del Anova, que existen diferencias estadísticamente significativas entre los grupos etarios estudiados tanto en la cantidad total de PSF como en cada tipo de ellos. Se realizó un análisis post hoc con la prueba de Tukey para conocer cuáles eran específicamente los rangos de edad que diferían entre sí.

Los resultados se presentan en la tabla 7 y en ellos se constató que todos los grupos diferían entre sí, tanto en el total de PSF como en los distintos tipos. Una situación particular se observa entre los 5 y 6 años que difieren en el total de PSF y luego, solo en los procesos de asimilación. 
Tabla 6

Comparación de la cantidad total de PSF y de los tipos de PSF entre los distintos rangos de edad

\begin{tabular}{lcccccccccc}
\hline Rangos de edad & \multicolumn{2}{c}{$\mathbf{3 - 3 , 1 1}$} & \multicolumn{2}{c}{$\mathbf{4 - 4 , 1 1}$} & \multicolumn{2}{c}{$\mathbf{5 - 5 , 1 1}$} & \multicolumn{6}{c}{$\mathbf{6 - 6 , 1 1}$} & Anova \\
\hline Tipos de PSF & $\mathbf{M}$ & $\mathbf{D E}$ & $\mathbf{M}$ & $\mathbf{D E}$ & $\mathbf{M}$ & $\mathbf{D E}$ & $\mathbf{M}$ & $\mathbf{D E}$ & \\
\hline Total de PSF & 27,45 & 13,58 & 19,21 & 13,45 & 8,67 & 7,95 & 5,88 & 6,49 & $93,728^{*}$ \\
Estructura & 14,35 & 8,71 & 9,71 & 7,82 & 4,14 & 4,34 & 2,64 & 3,55 & $76,677^{*}$ \\
Asimilación & 6,06 & 4,32 & 4,15 & 3,07 & 2,29 & 2,40 & 1,43 & 1,79 & $51,336^{*}$ \\
Sustitución & 7,04 & 4,51 & 5,35 & 4,87 & 2,23 & 3,17 & 1,81 & 2,22 & $47,808^{*}$ \\
\hline
\end{tabular}

${ }^{*} \mathrm{p}<.001$

$\mathrm{N}=112$ en cada grupo de edad

Tabla 7

Valores de la prueba de Tukey (diferencia de medias) y rangos de edad

\begin{tabular}{cccccc}
\hline PSF & Total de PSF & \multicolumn{3}{c}{ Tipos de PSF } \\
\cline { 1 - 1 } \cline { 5 - 6 } Rango de edad comparados & (Tukey) & Estructura & Asimilación & Sustitución \\
\hline $3,0-3,11$ vs $4,0-4,11$ & $8,24^{*}$ & $4,64^{*}$ & $1,91^{*}$ & $1,69^{*}$ \\
$4,0-4,11$ vs $5,0-5,11$ & $10,54^{*}$ & $5,56^{*}$ & $1,86^{*}$ & $3,12^{*}$ \\
$5,0-5,11$ vs $6,0-6,11$ & $2,79^{*}$ & 1,50 & $0,87^{*}$ & 0,42 \\
$3,0-3,11$ vs $5,0-5,11$ & $18,78^{*}$ & $10,21^{*}$ & $3,77^{*}$ & $4,80^{*}$ \\
$3,0-3,11$ vs $6,0-6,11$ & $21,56^{*}$ & $11,71^{*}$ & $4,63^{*}$ & $5,22^{*}$ \\
$4,0-4,11$ vs $6,0-6,11$ & $13,32^{*}$ & $7,06^{*}$ & $2,72^{*}$ & $3,54^{*}$ \\
\hline
\end{tabular}

* $p<0.05$

$\mathrm{N}=112$ en cada grupo de edad

En síntesis, los análisis anteriores contribuyen a ratificar la validez del Teprosif-R, puesto que es sensible para discriminar entre los distintos grupos etarios.
DISCUSIÓN

El Teprosif-R (Pavez, Maggiolo \& Coloma, 2008) evalúa los procesos de simplificación fonológica en niños de 3 a 6 años, los cuales están clasificados 
en tres grandes grupos según los procesos que afectan la estructura de la palabra, procesos de sustitución y procesos de asimilación. En este contexto, teniendo en cuenta que en nuestro país no contamos con un test que evalúe lo mencionado se procedió a adaptar psicométricamente el Teprosif-R (2008). En primer lugar, se evaluó la validez, a través de un juicio de expertos, quienes consideraron que la prueba tiene validez de contenido, ya que contribuye a lograr el objetivo de la investigación, las instrucciones son fáciles de seguir, está organizado en forma lógica, el lenguaje utilizado es apropiado al público al que va dirigido, existe coherencia entre variables, indicadores e ítems, y que sus reactivos representan el constructo de procesos de simplificación fonológica y su relación con los rangos de edad.

Al comparar los procesos de simplificación fonológica con la edad, se encuentra que los resultados hallados son similares a lo encontrado en la versión chilena (2008), que también hallan una relación significativa entre la edad y los procesos de simplificación fonológica, lo cual evidentemente está de acuerdo con la teoría y la psicología evolutiva desde el punto de vista psicolingüístico; vale decir que en un ser humano normal, a medida que incrementa su edad, los procesos simplificadores disminuyen, es decir que, si hallamos procesos simplificadores en una edad que no corresponde debemos recomendar una evaluación más exhaustiva en cuanto a esta variable. Estos resultados nos 1levan a concluir que la prueba Teprosif-R, al igual que en otras realidades es válida para la medición de los procesos de simplificación fonológica.

La confiabilidad se estimó calculando el coeficiente de consistencia interna alfa de Cronbach, cuyo valor de 0.94 es bastante elevado, lo cual resulta similar a lo hallado en la versión chilena (2008). También se calculó la correlación del ítem con el puntaje total, con resultados que indican que todos los ítems son altamente significativos, siendo el promedio de las correlaciones superiores a 0.50 , lo que significa que los ítems tienden a conformar una dimensión.

Por otro lado, la estimación de los niveles de dificultad corrobora que a medida que se incrementa la edad, el grado de dificultad de los ítems es menor, resultados que coinciden con los hallados en la versión chilena.

Con referencia a las normas de calificación, los baremos construidos también en función de los resultados hallados permiten la calificación de normal, riesgo y déficit, lo cual nos permite hacer una mejor observación del rendimiento de los niños.

En el presente trabajo, en comparación con la versión chilena que cuenta con 37 ítems, luego del análisis de estos se tiene un total de 40 ítems. En la inclusión de 3 nuevos ítems, se consideraron fonemas y grupos consonánticos no evaluados en la versión original; en 
la adaptación lingüística de 7 ítems a nuestro contexto sociocultural, se tomó en cuenta la estructura silábica, la acentuación y la metría de los ítems originales; y en la adaptación gráfica de 22 ítems se realizó con el fin de mejorar la percepción visual.

\section{CONCLUSIONES}

- Se logró la adaptación lingüística y pictórica del Teprosif-R a la realidad peruana para el uso en la evaluación de los procesos de simplificación fonológica en niños y niñas de 3 a 6 años de edad.

- El Teprosif-R muestra evidencias de validez de contenido, concurrente y de constructo.

- El Teprosif-R adaptado tiene una buena confiabilidad estimada por medio de la técnica de consistencia interna mediante el coeficiente alfa de Cronbach (0.94).

- Se determinaron los niveles de dificultad de los reactivos del Teprosif$\mathrm{R}$ clasificados en fácil, mediano, difícil y muy difícil.

- Se obtuvieron normas en percentiles para interpretar los niveles de desempeño según el total de PSF y sus tipos en cada rango de edad, considerando el test completo.

- Se estimó la confiabilidad y validez, y se calcularon las normas de los 15 primeros ítems del Teprosif-R que corresponden al screening.

\section{REFERENCIAS}

Acosta, V., León, S., \& Ramos, V. (1998). Dificultades del habla infantil: Un enfoque clínico. Investigación, teoría y práctica. Málaga: Ediciones Aljibe.

Acosta, V., \& Moreno, A. (2001). Dificultades del lenguaje en ambientes educativos. Del retraso al trastorno especifico del lenguaje. Madrid: Ediciones Barcelona.

Acosta, V., Moreno, A., Ramos, V., Quintana, A., \& Espino, O. (1996). La evaluación del lenguaje. Teoría y práctica del proceso de evaluación de la conducta lingüistica infantil. Madrid: Aljibe.

Alarcón, R. (2008). Métodos y diseños de investigación del comportamiento. Lima: Universidad Ricardo Palma.

Aliaga Tovar, J. (2006). Psicometría: test psicométricos, confiabilidad y validez. En: Quintana, A., \& Montgomery, W. (eds.). Psicología: Tópicos de actualidad. Lima: Universidad Nacional Mayor de San Marcos, 85-108.

Balluerka, N., Gorostiaga, A., AlonsoArbiol, I., \& Haranburu, M. (2007). La adaptación de instrumentos de medida de unas culturas a otras: una perspectiva práctica. Psicothema, 1(19), 124-133. Universidad del País Vasco. Recuperado de http://www. psicothema.com/pdf/3338.pdf 
Bosch, L. (2004). Evaluación fonológica del habla infantil. Barcelona: Masson.

Cervera, J., \& Ygual, A. (2003). Intervención logopédica en los trastornos fonológicos desde el paradigma psicolingüístico del procesamiento del habla. Revista de Neurología, 36(1), 39-53. Recuperado de http://cvu.rediris.es/pub/bscw.cgi/ d344458/Int\%20tras\%20fonol.pdf

Consejo General de Colegios Oficiales de Psicólogos. Traducción-adaptación de los test. Directrices para la traducción-adaptación de los test. Adaptadas de la Internacional Test Commission (ITC). Recuperado de www.cop.es/vernumero.asp?id $=42$

Cuetos, F. (2008). Psicología de la lectura. Madrid: Wolters Kluwer.

Drockell, J., \& Mc Shine, J. (1997). Dificultades del aprendizaje en la infancia. Un enfoque cognitivo. Barcelona: Paidós.

Hernández, R., Fernández, C., \& Baptista, P. (2010). Metodología de la investigación. México, D.F.: McGraw-Hill.

Holguín, M. (2008). Adaptación del test $A B C$ de Filho en niños bilingües de las comunidades de Pisac, Cusco. Tesis de Maestría. Lima: Pontificia Universidad Católica del Perú.

Ingram, D. (1983). Trastornos fonológicos en el niño. Barcelona: Editorial Médica y Técnica.
Jakobson, R. (1974). Lenguaje infantil y afasia. Madrid: Ayuso.

Melgar, M. (1976). Cómo detectar al niño con problemas del habla. México, D. F.: Trillas.

Ministerio de Educación de la República del Perú. Unidad de Medición de la Calidad Educativa (2004). Una aproximación a la alfabetización lectora de los estudiantes peruanos de 15 años. Resultados del Perú en la evaluación internacional PISA. Recuperado de www.oei.es/quipu/ peru/lectura_pisa.pdf

Monfort, M., \& Juárez Sánchez, A. (1989). Registro fonológico inducido. Madrid: Cepe.

Montero, C. (2005). Estrategias para afrontar la emergencia educativa: Experiencia y opciones. Lima: Instituto de Estudios Peruanos. Recuperado de www.oeiperu.org/documentos/CAF_Informe_Peru.pdf

Muñiz, J. (1996). Psicometría. Madrid: Universitas.

Pávez, M., Maggiolo, M., \& Coloma, C. (2008). Test para evaluar los procesos de simplificación fonológica Teprosif-R. Santiago: Ediciones Universidad Católica de Chile.

Peña, G., Cañoto, Y., \& Santalla de Banderali, Z. (2006). Una introducción a la psicología. Caracas: Texco.

Piñeiro, A., Manzano, M., Inguanzotsa, G., Reginosa, V., Morales, A., \& 
Fernández, C. (2000). Adaptación y normación de la prueba de vocabulario Peabody en sujetos cubanos. Revista Cubana de Psicología, 1(17). Recuperado de http://pepsic.homolog.bvsalud.org/scielo. php?script $=$ sci_arttext\&pid=S025743222000000100009\&lng=es\&nrm $=$ iso

Quintana, A., \& Montgomery, W. (eds.) (2006). Psicología: Tópicos de actualidad. Lima. Universidad Nacional Mayor de San Marcos. Recuperado de www.unmsm.edu.pe/psicologia/.../Libro\%20EAPIntro.pdf
Rado, J. (2006). Adaptación del test de Boston a la población afásica que acude al INR. Tesis para obtener el grado de magíster. Lima: Pontificia Universidad Católica del Perú, Centro Peruano de Audición y Lenguaje.

Sánchez, C. H., \& Reyes, M. C. (2006). Metodología y diseños de la investigación científica. Lima: Visión Universitaria.

Santiuste, V., \& Beltrán, J. (1998). Dificultades de aprendizaje. Madrid: Síntesis.

Tornimbeni, S., Pérez, E., \& Olaz, F. (2008). Introducción a la psicometría. Buenos Aires: Paidós. 